\title{
FEA of Plastic Flow in AZ63 Alloy during ECAP Process
}

\author{
R. $\mathrm{KOCICH}^{a, *}$, M. KuRSA ${ }^{b}$ AND A. MACHÁČKOVÁ ${ }^{c}$ \\ ${ }^{a}$ Department of Materials Forming, Faculty of Metallurgy and Materials Engineering \\ VŠB TU Ostrava, 17. listopadu 15, CZ 70833 Ostrava-Poruba, Czech Republic \\ ${ }^{b}$ Department of Non Ferrous Metals Refining and Recycling, Faculty of Metallurgy and Materials Engineering \\ VŠB TU Ostrava, 17. listopadu 15, CZ 70833 Ostrava-Poruba, Czech Republic \\ ${ }^{c}$ Department of Thermal Engineering, Faculty of Metallurgy and Materials Engineering \\ VŠB TU Ostrava, 17. listopadu 15, CZ 70833 Ostrava-Poruba, Czech Republic
}

\begin{abstract}
Magnesium alloys based on $\mathrm{Mg}-\mathrm{Al}-\mathrm{Zn}$ system are very attractive materials in many industries due to their specific properties. One of common forming manners suitable for this kind of material is the application of severe plastic deformation method. This paper is focused on the deformation behavior of AZ63 alloy at equal channel angular pressing process in the temperature range of $250-320^{\circ} \mathrm{C}$. For this purpose finite element analysis was used. Main attention was devoted to the influence of processing parameters on the material flow. Among other, changes of the flow during equal channel angular pressing in chosen regions of the pressed samples were monitored. As variables, temperature, friction coefficient, strain rate, $\varphi$-angle or back pressure application were chosen. The comparison of the material flow lines at different conditions shows the affecting of material flow. There are obvious considerable differences between regions adjacent to the inner radius and those adjacent to the outer radius. For example in the case $\mu=0$ the dead zone is delimited by the angle $\Psi=36^{\circ}$, while in the case of friction given by the coefficient $\mu=0.04$, the value of the angle was $\Psi=26.5^{\circ}$.
\end{abstract}

PACS: 81.05.Bx, 81.20.Hy, 81.40.Lm, 81.40.Pq, 83.50.Ha

\section{Introduction}

The benefits of application of severe plastic deformation (SPD) have been known for a relatively long time, especially in the area of constructional metal materials. The main advantages of the SPD processes lie in their effect on mechanical properties of the processed materials [1-4] that result from the high refinement of the grains.

Probably the best researched SPD process is the equal channel angular pressing (ECAP). There is a relatively large number of written sources based on practical experiments and physical or numerical modeling in this sphere of the process.

A great deal of research was focused on the description of the geometry of the used die with regard to the angle between the particular sections of the channel $(\varphi)$ and the angle that defines the external radius of the generated channel $(\psi)$ or the homogeneity of the imposed strain $[5,6]$. However, an overwhelming majority of this research involved pure $\mathrm{Al}$ or its alloys. Compared with the mentioned group, other materials have been used to a far lower extent, such as for example $\mathrm{Mg}$, or its alloys. Although these materials have received growing attention over the past few years, especially thanks to their

\footnotetext{
* corresponding author; e-mail: radim.kocich@vsb.cz
}

specific properties, there are still areas that have to be defined more accurately to allow successful commercial application of SPD processes. It is known that in $\mathrm{Mg}$ alloys, a suitable forming method facilitates super-plastic behavior both at high strain rate as well as low temperatures $[7,8]$. A recently published article [9] brought interesting information on the concurrent growth of strength and plastic properties of the $\mathrm{Mg}$ alloy ZE41A after the application of ECAP when, by using a large number of passages, the yield strength increased by $120 \%$ and the ductility by $75 \%$. Like in the case of extrusion of $\mathrm{Mg}$ alloys in a multistage process [10], this significant growth of plastic properties is attributed to the activation of shear processes different from basal slip systems, or more precisely, further strain mechanism during ECAP.

It can generally be declared that more complicated deformation behavior can be anticipated in alloys with a low number of slip systems. For this reason, several strategic methods for successful processing of "difficult to deform" materials have been verified. One of the proposed solutions (aforementioned) suggests processing these materials at higher temperatures, thus increasing their formability thanks to the activation of non-basal slip systems [11]. This benefit, however, is limited by the undesirably large grain size after the pressing. The second approach to solving the low formability after ECAP is the application of dies with larger angles between indi- 
vidual sections of the channel. There have been cases when the material was segmented after being pressed through a die at the angle of $90^{\circ}$, but if the material was pressed through the die at a larger angle, several passages were performed without disrupting its integrity [12]. Another example, the study [13], indicates that the pressing of an $\mathrm{Mg}$ alloy (ZK60) through a die at an angle of $90^{\circ}$ caused the creation of cracks along the entire length of the sample, pressing through a die at an angle of $110^{\circ}$ produced trouble-free pressing (without visible cracks). The disadvantage of this method is a relatively low amount of embedded deformation in an every single passage. This fact brings about not only the need for a larger number of passages, but also the material losses of the sample. The third, frequently proposed solution lies in the application of back pressure (BP) as an additional tool for material failure suppressing [14]. However, as partial results suggest, even this effect cannot completely eliminate the emergence of cracks or other forms of failure, although they are greatly suppressed [15].

As mentioned above, the multistage process of extrusion is one of the well-proven processes in ECAP. Its effect has been experimentally confirmed especially with $\mathrm{Mg}$ alloys when usually the first or the following passages proceed at higher temperatures and the final passages at lower temperatures. A further option that offers more favorable behavior of materials on the $\mathrm{Mg}$ basis is a combination of conventional and unconventional methods. The grain size decreased and the super plastic properties increased in the initial pressing of $\mathrm{Mg}$ during a proposed process called EX-ECAP, which can serve as a concrete example [16, 17].

We must also mention the stability of a plastic flow of material as a factor that plays an important role in the ECAP process. Together with other factors, the plastic flow of the material affects both the magnitude of the imposed strain and its homogeneity and, last but not least, the disposition of the processed material to the development of cracks. The plastic flow has been researched by several authors who checked various methods of evaluation from theoretical determination [18] through experimental tests [19] up to utilization of numerical modeling which probably includes the largest number of published works especially as regards application of the finite element method (FEM) [15, 20]. Most of the published works consider absence of friction as an initial condition in the process and are most frequently executed for the angle $\varphi=90^{\circ}$. Just a small number of experiments describe strain behavior in the application of dies under the angle of $\varphi<90^{\circ}$.

The submitted article is concerned with the definition of strain behavior of magnesium alloys based on the $\mathrm{Mg}-\mathrm{Al}-\mathrm{Zn}$ system (specifically the AZ63 alloy) during ECAP when dies with various angles $\varphi$ are applied. In addition to the temperature and stress properties, the main research factors regarded the stability of the plastic flow of the material during ECAP at specified temperatures and other variable factors.

\section{Experimental}

Although there are partial studies focused on the description of material flow during the ECAP process, the validity of their outcomes is limited by certain simplifying circumstances (e.g. absence of friction, application of $2 \mathrm{D}$ conditions for deformation, etc.) [21, 22]. That is why the performed experiment resulted in prediction of plastic flow of the AZ63 alloy by the application of FEM and also mapping the strain behavior during an ECAP process. Forge 2009 software was used for FEM simulations. The numerical simulations monitored the behavior of the mentioned alloy in dies defined by angles $\left(30^{\circ}, 60^{\circ}\right.$, and $\left.90^{\circ}\right)$. As regards variable factors, different speed of extrusion $(5 \mathrm{~mm} / \mathrm{s}$ and $10 \mathrm{~mm} / \mathrm{s})$ was defined together with two temperature values at which ECAP proceeded $\left(250^{\circ} \mathrm{C}\right.$ and $\left.320^{\circ} \mathrm{C}\right)$ as well as the size of the Coulomb friction coefficient specified by the values 0.2 and 0.04. The numerical modeling also monitored the effect of back pressure as a potential tool for suppressing the instability of the plastic flow. The AZ63 alloy composed of ( $5.92 \mathrm{wt} \% \mathrm{Al} ; 2.69 \mathrm{wt} \% \mathrm{Zn} ; 0.21 \mathrm{wt} \% \mathrm{Mn}$; Mg balance) served as an experimental material. The material behavior of the AZ63 alloy was defined by the relations of stress-strain obtained from experimental tests [23]. The results were processed with a view to distinguishing differences of the mapped factors in particular parts of the processed material. For this reason, the graphic dependences of the monitored values were researched in the defined areas of the extruded sample. All places were situated on a plane passing through the center of the sample, parallel with its longitudinal axis. The accurate positions of these places are given in Fig. 1a; the same areas were selected for all the simulated versions.
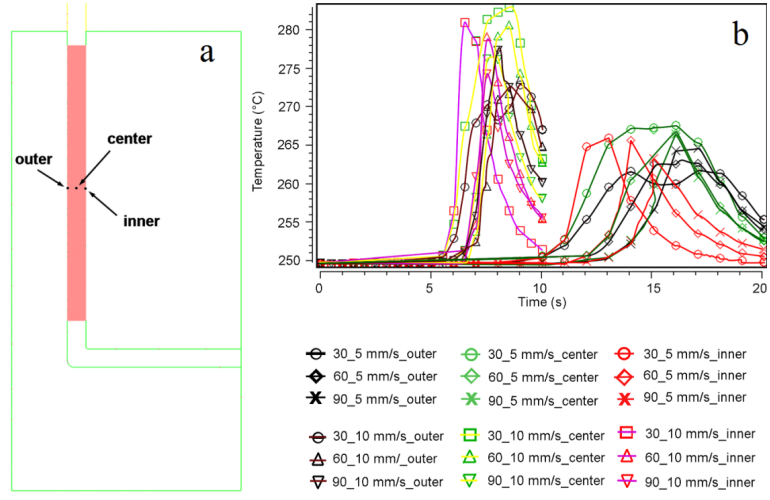

Fig. 1. (a) Distribution of monitored places. (b) Temperature in monitored places of AZ63 alloy during ECAP at $250^{\circ} \mathrm{C}$.

\section{Results and discussion}

After the evaluation of numerical simulations and from the enclosed results (Fig. 1b) it is clear that the mentioned temperature dependences present a relatively 
large difference in the peak temperatures. The applied higher extrusion velocity caused a growth of the maximum temperature values in all the mapped cases. It must be stressed that the largest increase of temperature caused by the development of deformation heat and friction was detected in a die with the angle of $\varphi=30^{\circ}$. Contrary to the lower applied extrusion velocity $(5 \mathrm{~mm} / \mathrm{s})$, the difference of the temperature was greater than $20^{\circ} \mathrm{C}$. It thus may be declared that a higher extrusion velocity generated a shorter total extrusion period; nevertheless, it caused a significant increase of temperature in the processed material. The second simulated case, i.e. ECAP at the temperature of $320^{\circ} \mathrm{C}$ showed very similar results. As regards higher temperature, the differences between both applied extrusion velocities were even higher (the detected difference reached up to $38^{\circ} \mathrm{C}$ ). As certain experimental studies suggest [24] the temperature at the start of the dynamic re-crystallization in $\mathrm{Mg}$ alloys may fluctuate within the range of $(150-330)^{\circ} \mathrm{C}$ according to the particular conditions. The results of the simulation thus prove the emergence of suitable thermal conditions for a potential commencement of dynamic re-crystallization of the processed alloy even if it is extruded at lower temperatures. These processes can thus occur as a result of increased temperature caused by the higher extrusion velocity, although the initial temperature of the extruded material is lower than the temperature necessary for the start of re-crystallization.

The evaluation of imposed strain preceded much like the evaluation of temperature dependences. The graphic layout of the imposed strain along the section of the extruded sample comes from a plane passing through its center. As Fig. 2a-c suggests, the value of the maximum imposed strain drops with the increased angle between the individual sections of the channel. The drop of homogeneity in the imposed strain along the section of the deformed sample is also obvious. The differences described in detail are also visible in Fig. 2d, which shows the progress of the imposed strain during the process in the monitored areas. With the growing $\varphi$ angle, the largest values of the imposed strain "move" towards the top surface of the extruded material. Dies with lower angles $\left(30^{\circ}\right.$ and $\left.60^{\circ}\right)$ are characterized by "dead zone" suppression similar as in the case of the die with the angle of $75^{\circ}$ published in [25]. The presence of the corner zone is confirmed only in the die with the angle of $90^{\circ}$. This dead zone is situated into an area adjoining the outer radius. Besides this corner zone, we must mention the areas where the material adheres imperfectly, mainly to the surface of a channel adjoining the internal radius (lateral gaps). These zones can be observed in higher as well as in lower extrusion velocities.

Similar outcomes have been published earlier [26, 27] but at that time the influence of the strain rate sensitivity $(m)$ on the formation of these lateral gaps in the outlet section of the channel was monitored. It has been proved that with a drop of the $m$ value, the cross-section of the pressed sample gradually decreases so that the sample

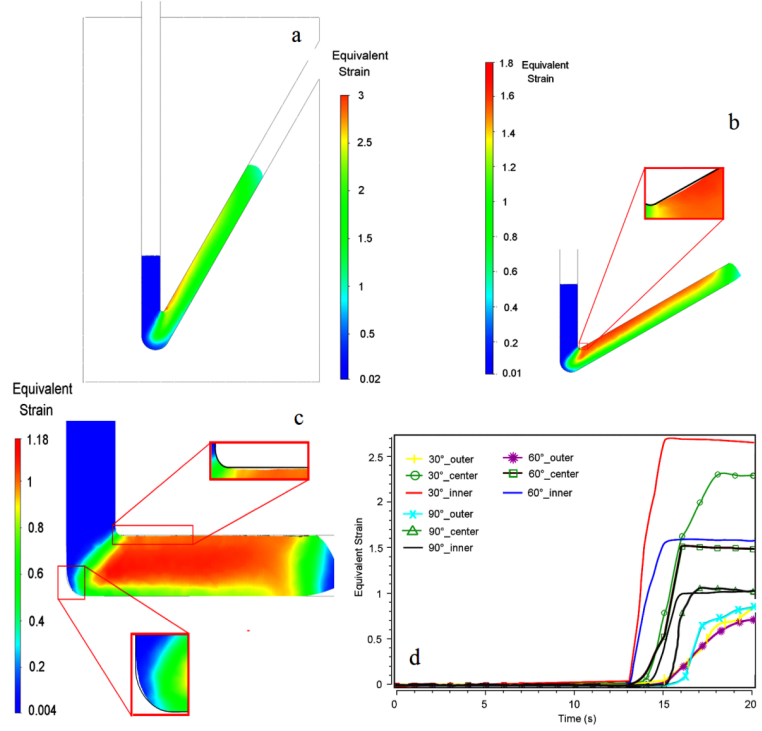

Fig. 2. (a)-(d) Equivalent strain for dies with various channel angles $(\varphi)$.

cannot perfectly fill a section of the outlet part of the channel. This fact results in formation of lateral gaps between the channel and the processed material, that initiate the rotation of the sample ends. The lateral gaps thus increase the non-homogeneity of the imposed strain and thus they are extending with the growing $m$ value. An effect identical with the growing $\varphi$ angle on a shift of the maximum imposed strain towards the top surface of the pressed material has even been detected for the rising $m$ coefficient. An increase of the $m$ value increases the values that determine the likelihood of the material failure. It also prompts a shift of these values to areas adjoining the inner corner of the channel (top layers of the material). This fact is attributed to the enlargement of the main deformation zone (MDZ) towards the outlet channel where tensile strengths are already present and contribute to the failure creation [13].

As regards the plastic flow of the processed alloy, it may be declared that during ECAP at the temperature $320^{\circ} \mathrm{C}$ (Fig. 3), there are certain differences compared with the conditions during the strain at the temperature of $250^{\circ} \mathrm{C}$ (Figs. 4, 5). The basic features include a tendency of the material to increase the length of areas that do not adhere to the top wall of the die channel (lateral gaps) with lowered temperatures. This tendency is also evident in relation to the growing extrusion velocity when, especially in the case of a die under the angle of $90^{\circ}$, the relative length of imperfect contact of the material with the top surface of the die increases. This feature is probably caused by higher plasticity of the material at higher strain temperatures. The supplemented grids also show a more desirable material flow in the case of a higher extrusion velocity. Besides the lower "wrinkling" of the horizontal lines of the superimposed grid, there 


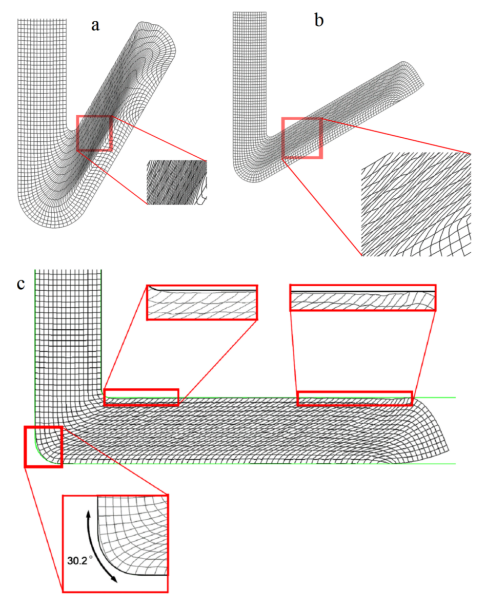

Fig. 3. (a)-(c) The predicted distortion of grid lines superimposed on the billet for various die angles under conditions of: $T=320^{\circ} \mathrm{C}, \mu=0.04, v=5 \mathrm{~mm} / \mathrm{s}$.

is a narrower transitional region between the areas of a very intensive slide and areas with a lower strain (adjoining the bottom surface of the material). A higher applied temperature had an analogous effect on the material flow. The details show that, in the application of ECAP at a lower temperature, a narrower area with a lower shearing strain than in the extrusion at higher temperatures was detected. The presented sequence of pictures confirms the mentioned non-homogeneity of the imposed strain along the section, particularly in dies with a smaller angle. A relatively small imposed shearing strain reaching the bottom half of the sample is evident; in both cases, it mostly concerns a bend. The top half of the extruded alloy shows very high shearing strains that are characterized by a relatively stable plastic flow of the material. It is also clear that, with the growing angle of the channel $(\varphi)$, the "rotation of free ends" (effect of ends) weakens. In a multiple extrusion, this detected phenomenon is responsible for the lower share of the imposed shear strain and its non-homogeneous distribution and can be one of the factors responsible for folds formation [28].

A mutual comparison of a plastic flow in the die with the angle $\varphi=90^{\circ}$ at the same velocity but a different temperature results in slowing the flow of layers in the bottom half of the samples when the temperature of the simulated process is lower $\left(250^{\circ} \mathrm{C}\right)$. It must be pointed out that this difference is only obvious in areas beyond MDZ. This detected drop probably coincides with a lower influence of friction at higher forming temperatures and better plasticity of the alloy at higher temperatures. This also prompts a growth of the dead zone when extruding at higher temperatures. The achieved shapes of individual cells of the grid suggest that the higher extruding speed resulted in lower shear strains imposed into the bottom areas of the sample.

According to the law regarding movement of individual metallic particles through a passage with the lowest

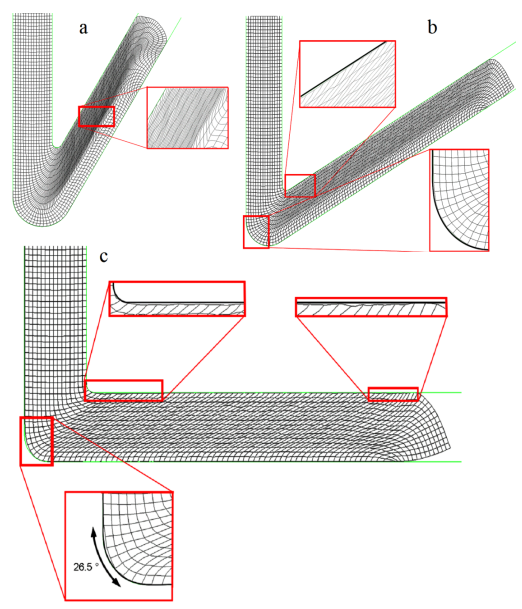

Fig. 4. (a)-(c) The predicted distortion of grid lines superimposed on the billet for various die angles under conditions of: $T=250^{\circ} \mathrm{C}, \mu=0.04, v=5 \mathrm{~mm} / \mathrm{s}$.

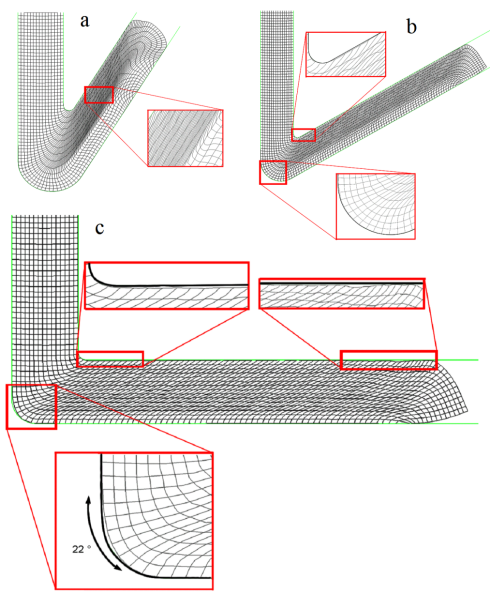

Fig. 5. (a)-(c) The predicted distortion of grid lines superimposed on the billet for various die angles under conditions of: $T=250^{\circ} \mathrm{C}, \mu=0.04, v=10 \mathrm{~mm} / \mathrm{s}$.

resistance, partial influences affecting the resulting plastic flow must be considered. The stability of the plastic flow in the ECAP process is thus one of the basic indicators determining the homogeneity of the resulting distribution of the imposed strain as well as the tendency towards cracks or fractures [18]. The plastic flow of material (its instability) serves as a basis for individual criteria defining its potential failure (Latham-Cockroft, Oyane) [19]. The tendency towards breaking the material during ECAP strongly depends on the size of oscillation in the processed material. Areas with the highest oscillation can produce concentration of stress which results in cracks formation. Most of the criteria are determined as an integral temporal relation of stress and strain. Among others some of mentioned criteria are constructed on the consideration of maximal principal stress evaluation.

Some of the processed alloys usually experience an occurrence of these failures in the form of cracks at the 
top surface of the sample. As the experimental findings suggest, using of material with large initial grain size in ECAP causes a very frequent gain in bimodal distribution of the grain size in the structure. Conversely, if the initial grain size is reduced (e.g. by a previous extrusion), a gain of an equiaxed fine-grained structure can be anticipated. The rising cracks are also greatly connected with the $m$ value. A higher $m$ coefficient usually causes only very shallow superficial cracks. Conversely, a low $m$ value causes segmentation (deep cracks along the entire cross-section of the sample).

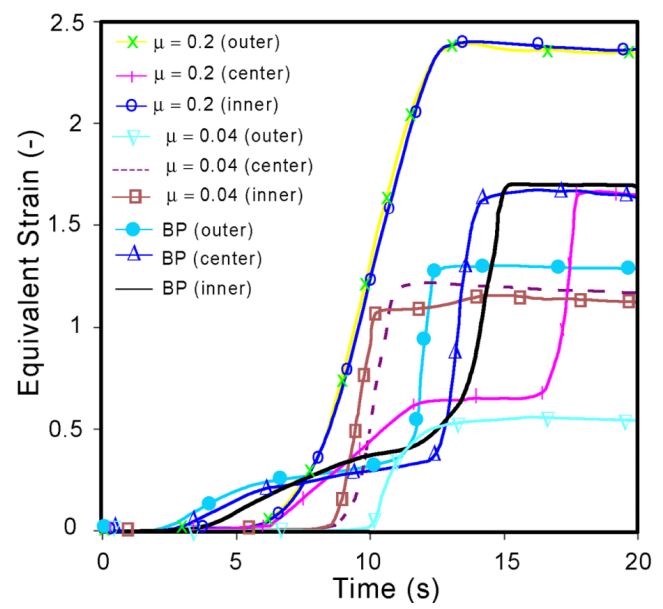

Fig. 6. Dependence of the imposed strain on the friction coefficient.

As regards a variable friction coefficient, the supplemented graphic relation (Fig. 6) provides observation of a sharp growth of the imposed strain during extrusion in increased friction $(\mu=0.2)$ up to twofold values in comparison with the lower modeled friction $(\mu=0.04)$.

While higher friction evidently led to higher imposed strain in areas adjoining the outer radius of the channel, the central areas show more gradual growth of the imposed strain. The ensuing growth of strain in the central areas is most likely caused by a growing friction which results from the growing length of the contact area between the channel and the surface of the sample in the course of time. This factor gradually builds up a resistance against the flow of the surface layers and, conversely, tends to support the central areas. In accordance with these findings there is also marked drop of the dead zone size in the case of higher friction. The increased friction thus favorably affects homogeneity of the strain during ECAP, particularly in the bottom half of the sample. This positive effect is, however, compensated by a necessity of higher working pressures (load on punch), which increases the danger of its damage.

As regards the lower friction coefficient, it can be declared that, unlike high friction, the lowest strain is reached in the bottom areas and maximum in the central and surface areas of the sample. An explanation of this fact may lie in the absence of the dead zone in case of the high friction. As for the lower friction, there are areas in the channel where the material does not tightly adhere to the walls of the channel, which of course affects the shape, or rather the location of MDZ. Due to the different location (shape) of MDZ, the resulting heterogeneity of the strain imposed in the ECAP process is also affected. The friction, together with a different applied speed and temperature of the process, affects the resulting size (shape) of the dead zone as well as the zones beyond MDZ (in the exit section of the channel). Besides these mentioned factors, the $m$ value can largely affect the size as well as the position of the MDZ. In practice, the extent of the failure is affected mainly by the width of the deformation zone.

The localization of slip into very thin zones prompts a higher inclination towards formation of cracks. A growing angle between the channels as well as the lowered $m$ value reduce the likelihood of a failure. A similar result appears with higher pressing speeds, which mean a higher risk of cracks formation. This finding has been confirmed by a practical experiment in $\mathrm{Ti}$ processing [11]. It must be stressed that higher extrusion velocity in our experiment caused an increase of strain rate in MDZ and above all their enlargement from the inner radius to the outer corner (not shown here).

As the mutual comparison of particular grids showed, higher extrusion velocity resulted in a lower resulting size of the dead zone. The size of the dead zone was largest $\left(30.2^{\circ}\right)$ for extrusion at $320^{\circ} \mathrm{C}$, the smallest size $\left(22^{\circ}\right)$ was reported for extrusion at $250^{\circ} \mathrm{C}$ (higher extrusion velocity). On the other hand, however, a better fill of the dead zone does not automatically mean higher uniformity of the imposed strain, as the study [29] has proved. For example the rise of the $m$ value means a reduction of the dead zone, but also a reduction of the lateral gaps in the outlet part of the channel. It is also true that identical friction does not produce an identical effect at various temperatures in the pressing, as became clear in the described experiment. Thus it can be declared that both friction and the $m$ value significantly affect the compressive component of deformation (i.e. uniformity of imposed strain).

In connection with the mentioned danger of failure creation, application of back pressure is frequently mentioned as one of the options eliminating these effects $[14,30,31]$. The next part of the experiment monitored the effect of the BP on the elimination of oscillation of the superimposed grid characterizing the plastic flow of material. This back pressure was defined by the value of $70 \mathrm{MPa}$ in a die with an angle of $90^{\circ}$.

A beneficiary effect of the back pressure was proved, particularly in a growth of the imposed strain. In addition, application of back pressure slightly homogenized the distribution of strain along a section of the extruded material (Fig. 6). However, it is clear that even the application of back pressure did not completely equalize the values of the imposed strain along the entire section and there are still certain differences in the size of the im- 


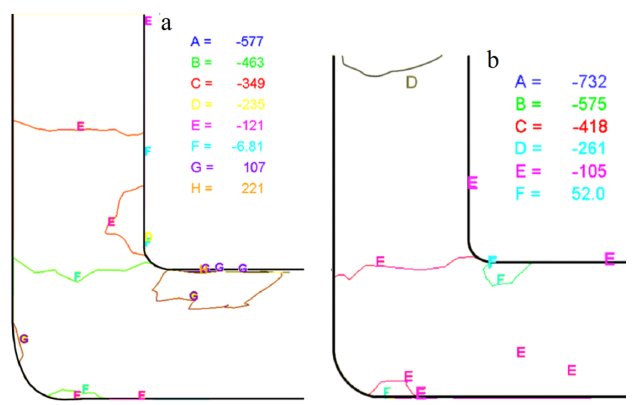

Fig. 7. Distribution of maximal principal stress (a) without $\mathrm{BP}$, (b) with $\mathrm{BP}$.

posed strain along the section of the processed material. Like in the lower friction, in BP the smallest imposed strain affects the bottom parts of the sample (adjoining the outer radius).

It should be stressed that BP caused apparent decrease of tensile stresses in the areas adjoining the inner radius (Fig. 7). This result will suppress the tendency to cracks formation in mentioned regions of the sample. It means the BP changes the positive sign of the maximal principal stress into the negative values (compressive character).

The positive factors accompanying the utilization of the back pressure thus include particularly the growth of the imposed strain together with its certain homogenization; however, like in the high friction, there is a relatively high growth of the working load to extrude the sample through the die similar to that for the dies with a smaller angle (Fig. 8). When evaluating the effect of the $\mathrm{BP}$, it must be stressed that although it completely eliminated the existence of the dead zone (similarly as in the case of higher friction), it did not eliminate the instability of the plastic flow as is confirmed by serrations in the punch load-time curve. In the case of unstable flow, the strain heterogeneity is caused by shear concentration localized along layers of softer material. In spite of the positive influence of BP on dead zones suppression, it does not have any influence on the formation of softer layers in the processed sample. Due to this reason, shear concentrations (unstable flow) will develop despite the $\mathrm{BP}$ utilization. The described relation also suggests that the simultaneous effect of back pressure and high friction does not produce a very favorable effect as regards the attained homogeneity of deformation. A version with a "medium" friction value in a simultaneous BP application appears far more ideal.

As it was mentioned before, the usage of material with coarse initial grain size causes a very frequent gain in bimodal distribution of the grain size in the structure. Conversely, if the initial grain size is low (e.g. by a previous pressing), a gain of an equiaxed fine-grained structure can be anticipated [13]. Nevertheless, for successful processing of "hard to deform" materials (Mg alloys, particularly based on $\mathrm{Mg}-\mathrm{Al}-\mathrm{Zn}$ ), it is necessary to observe all the mentioned factors and the morphologic type of

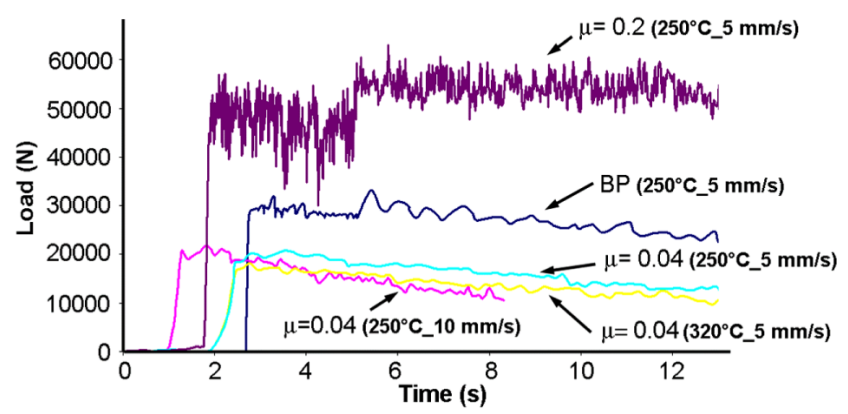

Fig. 8. Obtained loads on punch during ECAP process under different conditions.

the given microstructure. This is because the main factors include inter-metallic phases or more precisely the pattern of their appearance and so forth.

\section{Conclusion}

The deformation behavior of the AZ63 alloy during an ECAP process was modeled by application of FEM simulations. The results clearly show that small angles may bring higher stability of the plastic flow, but at the expense of high non-homogeneity of the imposed strain along the cross-section of the extruded material. The highest homogeneity of the strain was detected in a die with the angle of $90^{\circ}$. Lower angles of dies brought higher values of imposed strain similarly as higher friction coefficient. This factor is caused by perfect filling of the outer corner of the die (dead zone was decreased or removed). Nevertheless, usage of dies with small angles increases the likelihood of a failure creation. The main reason very probably lies in high shear deformations mainly in areas adjoining the inner corner of the channel. There are great differences between the flow in the top and bottom layers of the material despite the relatively stable material flow in these layers. The mentioned difference in material flow along the cross section of the sample as well as possible instabilities in material flow may generate undesired tensile stresses that are responsible for a potential failure.

Increasing friction tends to decrease the size of dead zone, so that this factor assures a complete filling of the outer corner by providing a concentrated shear in a very narrow zone, so that the deformation is larger. The application of back pressure brought higher homogenization of the strain, but did not eliminate the instability of the plastic flow. FEM simulations confirmed that an imposition of BP reduces the gaps between the billet and the die and thereby increases overall strain homogeneity on the cross-section of the billet. Great contribution of $\mathrm{BP}$ imposing is in the significant reduction of maximum principal stresses in the strained area of the billet. The tendency for cracking is suppressed in this way because it depends upon contemporary reaching of critical values of the amount of strain as well as the level of maximum principal stresses. 
With a rising temperature, the length of areas with bad adhesion of the extruded material, particularly to the top wall of the channel, is growing. Higher extrusion velocities resulted in higher values of oscillation in the material flow, i.e. means less favorable material flow and higher probability of stress concentration. As regards ECAP at a lower temperature, a narrower area with a lower shearing strain than in extrusion at higher temperatures was detected. With decreasing angle of the die channel or growing magnitude of the friction coefficient a higher punch pressure was needed.

As this article suggests, the reduction of pressing speed, use of dies with a larger angle or BP application may similarly like previously published factors (reduction of initial grain size or increase of the $m$ value) result in suppression of cracks in the ECAP process.

\section{Acknowledgments}

This paper was created in the project No. CZ.1.05/2.1.00/01.0040 "Regional Materials Science and Technology Centre" within the frame of the operation programme "Research and Development for Innovations" financed by the Structural Funds and from the state budget of the Czech Republic.

\section{References}

[1] M. Furukawa, Z. Horita, M. Nemoto, R.Z. Valiev, T.G. Langdon, Philos. Mag. A 78, 203 (1998).

[2] M.V. Markushev, C.C. Bampton, M.Y. Murashkin, D.A. Hardwick, Mater. Sci. Eng. A 234-235, 927 (1997).

[3] R. Kocich, M. Kursa, I. Szurman, A. Dlouhý, J. Alloys Comp. 509, 2716 (2011).

[4] R. Kocich, J. Fiala, I. Szurman, A. Macháčková, M. Mihola, J. Mater. Sci. 46, 7865 (2011).

[5] E. Hosseini, M. Kazeminezhad, Comput. Mater. Sci. 44, 962 (2009)

[6] N. Mahallawy, F.A. Shehata, M. Hameed, M.I. Aal, H.S. Kim, Mater. Sci. Eng. A 527, 1404 (2010).

[7] W.J. Kim, S.I. Hong, Y.S. Kim, S.H. Min, H.T. Jeong, J.D. Lee, Acta Mater. 51, 3293 (2003).

[8] M. Mabuchi, H. Iwasaki, K. Yanase, K. Higashi, Scr. Mater. 36, 681 (1997).

[9] A. Ma, J. Jiang, N. Saito, I. Shigematsu, Y. Juan, D. Yang, Y. Nishida, Mater. Sci. Eng. A 513-514, $122(2009)$.
[10] J.C. Tan, M.J. Tan, Mater. Sci. Eng. A 339, 124 (2003).

[11] S.L. Semiatin, V.M. Segal, R.E. Goforth, N.D. Frey, D.P. DeLo, Metall. Mater. Trans. 30A, 1425 (1999).

[12] M. Furui, H. Kitamura, H. Anada, T.G. Langdon, Acta Mater. 55, 1083 (2007).

[13] R.B. Figueiredo, P.R. Cetlin, T.G. Langdon, Acta Mater. 55, 4769 (2007).

[14] V.V. Stolyarov, R. Lapovok, I.G. Brodova, P.F. Thomson, Mater. Sci. Eng. A 357, 159 (2003).

[15] R.B. Figueiredo, P.R. Cetlin, T.G. Langdon, Metall. Mater. Trans. 41A, 778 (2010).

[16] Z. Horita, K. Matsubara, K. Makii, T.G. Langdon, Scr. Mater. 47, 255 (2002).

[17] Y. Miyahara, Z. Horita, T.G. Langdon, Mater. Sci. Eng. A 420, 240 (2006).

[18] V.M. Segal, Mater. Sci. Eng. A 345, 36 (2003).

[19] G.M. Stoica, D.E. Fielden, R. McDaniels, Y. Liu, B. Huang, P.K. Liaw, C. Xu, T.G. Langdon, Mater Sci. Eng. A 410-411, 239 (2005).

[20] R.B. Figuiredo, P.R. Cetlin, T.G. Langdon, Mater. Sci. Eng. A 518, 124 (2009).

[21] H.S. Kim, Mater. Sci. Eng. A 328, 317 (2002).

[22] R. Kocich, M. Greger, A. Macháčková, in: Proc. Conf. Metal, TANGER, Ostrava 2010, p. 166.

[23] S.E. Ion, F.J. Humpreys, S.H. White, Acta Metall. 30, 1909 (1982).

[24] A.V. Nagasekhar, T.H. Yip, S. Li, H.P. Seow, Mater. Sci. Eng. A 410-411, 269 (2005).

[25] H.S. Kim, S.I. Hong, M.H. Seo, J. Mater. Res. 16 , 856 (2001).

[26] J.W. Park, J.Y. Suh, Metall. Mater. Trans. 32A 3007 (2001).

[27] J.C. Werenskiold, H.J. Roven, in: Proc. Conf "Nanomaterials by Severe Plastic Deformation NANOSPD2", Vienna (Austria), 2002, p. 591.

[28] M.G. Cockroft, D.J. Latham, J. Inst. Metals 96, 33 (1968).

[29] R.K. Oruganti, P.R. Subramanian, J.S. Marte, M.F. Gigliotti, S. Amancherla, Mater. Sci. Eng. A 406, 102 (2005).

[30] F. Djavanroodi, M. Ebrahimi, Mater. Sci. Eng. A 527, 1230 (2010).

[31] P.W.J. Mckenzie, R. Lapovok, Y. Estrin, Acta Mater. 55, 2985 (2007). 